\title{
A Framework for Label Images
}

\author{
Loïc Mazo* \\ Université de Strasbourg, LSIIT, UMR CNRS 7005, France \\ loic.mazo@unistra.fr
}

\begin{abstract}
Label images need a specific topological model to take into account not only the topologies of the regions but also the topology of the partition. We propose a framework for label images in which all the regions of the initial partition and of any coarser partition of the space can be explicitly represented. Some properties of the model are given and a local transformation that preserves the weak homotopy types of all the regions of all the partitions is defined.
\end{abstract}

Keywords: Label image, simple point, homotopy type.

\section{Introduction}

In this paper, we study, from a topological viewpoint, digital label images, that is, images whose domain is $\mathbb{Z}^{n}$ and whose codomains are sets on which there generally exists no meaningful order relation (unlike grey-level images for instance). Label images need a specific approach in topology. Indeed, a label image is much more than a collection of independent objects and we are also interested in the spatial relations between these objects. Thereby, any topologically sound label image processing must pay attention to the objects and to the partition of the space associated to the labels. Nevertheless, as far as we know, the literature devoted to the topology of label images is not well developed and essentially oriented towards specific applications. The most commonly used approach is to process one label at a time while rejecting temporarily the other labels in the background, coming down to a binary image (e.g. 14/3/8 ). With this method, either the topology of the partition is ignored or it is necessary to adjoin another structure, like a region adjacency graph, with the possibility to lose some information on tunnels or knots. Even in the case where the configuration permits a binary treatment of the image (in 9]12 the objects are concentric topological spheres), there are some specific issues. In a binary image, one usually finds just one object of interest embedded in an ambient space without significance. Hence, the choice of the adjacency pair can be done accordingly to the (known or expected) properties of the unique considered object. Now, if the binary image is a label image, that is to say, the two members of the partition are regions of interest, how to decide the region that will be equipped with the 6 -adjacency and the

\footnotetext{
* The research leading to these results has received funding from the French Agence Nationale de la Recherche (Grant Agreement ANR-2010-BLAN-0205).
} 
one that will be equipped with the 26-adjacency relation for instance? In [12], the choice is made in relation with the nature of the objects (their thickness) and in 9 , the choice is made after algorithmic considerations. In both cases, that could be unsatisfactory if the two objects of interest are similar. Furthermore, most of the time, in the applications (see, e.g., [13]) the value of the image on a picture element changes from the background to a particular label (depending on a cost, or energy, function), or vice versa, but more rarely it goes from a label to another label (and it occurs that points cannot be labelled since any choice of a label would break an a priori knowledge or would create a forbidden configuration like a cross over). An important drawback of this method is that an object identified by a label is inevitably seen under distinct adjacencies at distinct times of the process and even the number of the connected components is no more an invariant. This problem can be solved by working in the class of well-composed images [7] which uses the same adjacency relation for the object and for the background (namely, the (2n)-adjacency relation). Moreover, it is possible to transform (in a non-injective manner) an ordinary label image into a well-composed image [17] but the algorithm needs an order on the labels. The approach proposed in 4 makes it possible to change the label of a point in a 3D cellular space by choosing a new label among several ones with the assurance that the homotopy types of the two labels, the new one and the former one, are preserved. Nevertheless, no attention is paid to the topology of the partition. To take this latter topology in consideration, it is required in 2 that the unions of two labels (in 2D spaces), or two or three labels (in 3D spaces), are watched in any process as well as single labels. However, a careful examination of the examples provided by the authors shows that these conditions are not sufficient to maintain the topology of the partition. In particular, the unions of three labels should also be watched in a $2 \mathrm{D}$ space. In [5], the authors study label images in $\mathbb{Z}^{3}$ with objects that are 6-connected. They understand the topology of the partition as the set of the topologies of the surfaces between 6 -adjacent labels. When performing a change of label on a point, they use simple points in the $(6,18)$ adjacency framework of $\mathbb{Z}^{3}$ to maintain both topologies of the thinned label and of the growing one, while they use collapses in the cubical complex framework to ensure the preservation of the topologies of the surfaces between 6 -adjacent labels. In a previous work [1], we have proposed an extensive theoretical framework to deal with label images. The main idea is that, topologically, a label image is a set of regions (that share the same label) together with some unions of these regions (thus, we retain the idea exposed in [2]). In other words, a label image is a partition together with some coarser partitions which are meaningful and a topologically sound processing of the image must control the topologies of all the regions of these partitions. In our framework, the label images are defined on a poset (partially ordered set), typically the space of cubical complexes equipped with the inclusion, and take their values on an atomisti 1 lattice of labels. The atoms of the lattice are the labels of the initial digital image (defined on $\mathbb{Z}^{n}$ )

${ }^{1}$ A lattice is atomistic if any element, but the minimum, is a supremum of atoms. 
and the supremum operation is used to create the unions of labels of interest (the coarser partitions). At least, besides the atoms, the lattice of labels must contain a minimum and a maximum (leading to the lattice of labels described in [15 16]). At most, the lattice is the power set of the atoms. In [11], we describe some transformations on label images that preserve the weak homotopy type of all the regions of interest. Nevertheless, this model has an important drawback at the first stage: the embedding of the digital space in a poset. If the digital objects are not modeled by closed subsets of $\mathbb{R}^{n}$ (i.e. if the adjacency relation for the objects is not the $\left(3^{n}-1\right)$-adjacency), the embedding cannot preserve the topology of the labels together with their unions as illustrated on Figure 1. To overcome this issue, we have been lead to develop another model that we describe in this article.

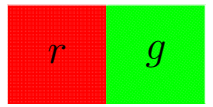

(a)

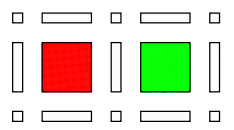

(b)

Fig. 1. (a) A digital image $\lambda$ in $\mathbb{Z}^{2}$ with 2 labels $r$ (red), $g$ (green) and a background (not depicted). (b) The embedding of the image $\lambda$ in $\mathbb{F}^{2}$, the space of cubical 2-complexes, obtained by applying the following membership rule: the label of a face is the infimum of the labels of the surrounding facets in the poset $\left(2^{\{r, g\}}, \subseteq\right)$. We have proved in 10 that this embedding preserves the connected components and the fundamental groups of the object and its complement when a binary digital image is interpreted with the $\left(2 n, 3^{n}-1\right)$-adjacency pair. But, if we identify the two labels, that is if we consider a coarser partition of the space, the topology is not the same on these two images (we have one component on the left and two components on the right).

The remainder of this article is organized as follows. In Section 2, we define the covering images, the kind of abstract image that we propose to model a label digital image. In Section 3, we expose a notion of simple point for covering images. In Section 4, we show that the classical duality for binary images (object/background) can be extended to covering images. Section 5 concludes this paper and indicates some results, more technical, that we cannot expose here by lack of space. Note that, also by lack of space, no proofs are given in this article.

\section{Covering Images}

In order to model all the topological relations that can be found in a digital label image $\lambda$ (defined on $\mathbb{Z}^{n}$ ), we propose two steps.

1. We split the image $\lambda$ in a collection of binary images that represent the regions of interest, that is the regions that have been labeled (for instance during a segmentation process) and a number of unions of these regions 
if we are interested in some inter-labels relations. The unions are labeled thanks to a lattice structure on labels: the label of an union of regions is the supremum of the labels of the regions. No other labels are needed for our purpose, so the lattice of labels, noted $T$, is an atomistic lattice (whose atoms are the initial labels) and there is a one-to-one correspondence between this lattice and the collection of binary images (we write $\lambda_{t}$ for the binary image associated to the label $t$ in the collection built from the digital label image $\lambda$ ). Figure 2 exemplifies this first stage (in the sequel, the infimum and supremum operators on $T$ are denoted $\wedge$ and $\vee$ while $\perp$ and $T$ are the minimum and the maximum of $T$ ).

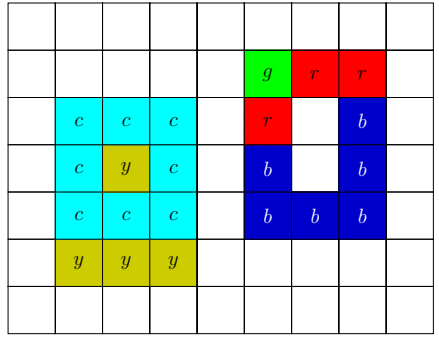

(a)

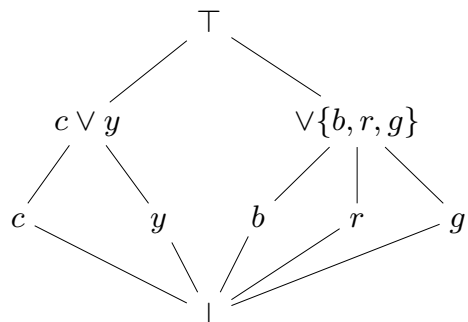

(b)

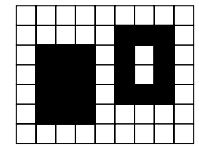

(c)

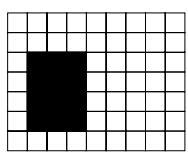

(d)

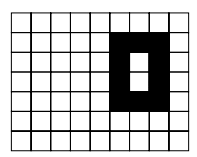

(e)

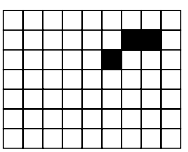

(i)

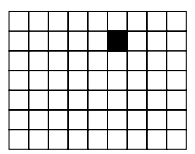

(j)

(f)

(g)

(h)

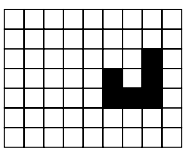

Fig. 2. (a) A digital label image with five labels $c, y, b, r, g$ respectively depicted in cyan, yellow, blue, red and green. (b) A lattice structure $T$ whose atoms are $c, y, b, r, g$. (c-j) The collection of binary images associated to the lattice $T$ (the binary image associated to $\perp$ is not represented for it is a constant image, with no object). 
2. In each binary digital image $\lambda_{t}$ created in the previous step, we introduce inter-xels elements (pointels, linels, surfels and so on) to be able to use topological tools. The space in which we embed the digital images is the space of cubical complexes, $\mathbb{F}^{n}$, but it could be another cellular decomposition of the space 2 . The inter-xels elements must be labeled with membership rules with respect to the desired interpretation of the image. Such rules can be found in the literature (see e.g. [1]). We have proposed in [10] our own rules which preserve the connected components for the classical adjacency pairs and result in isomorphisms between the digital fundamental groups as defined by Kong and the classical fundamental groups of the regions of $\mathbb{F}^{n}$. Note that distinct rules can be applied to distinct labels provided no inconsistency is introduced (for instance, if two voxels are connected in the region labeled $A$, they cannot be disconnected in the region labeled by $A$ or $B$ ): this leads us to the notion of fiber described below.

After these two steps have been achieved, we get a collection $\left(\mu_{t}\right)_{t \in T}$ (actually a lattice) of binary images, the sheets, defined on $\mathbb{F}^{n}$ (see Figure 3 and Figure 4). Now, we can see this collection as a unique image $\mu$ by setting that $\mu(x)$ is equal

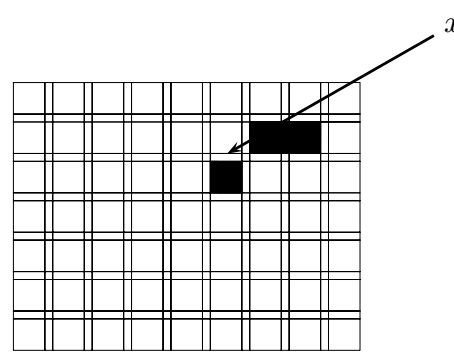

(a)

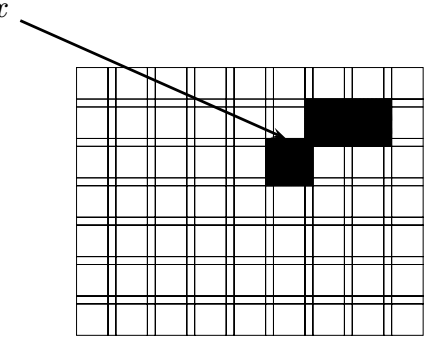

(b)

Fig. 3. The sheet associated to the label $r$ of the digital label image depicted on Figure 2 (a) The digital binary image of Figure 2(i) is interpreted with the $(4,8)$ adjacency pair (the object of the binary image is open, it does not include its boundary).

(b) The same image is interpreted with the $(8,4)$-adjacency pair (the object is closed so it includes its boundary).

to the set (we say the fiber) whose elements are the (extended) labels $t$ such that $\mu_{t}(x)=1$ (in other words, the labels attached to $x$, or, equivalently, the regions of interest $x$ belongs to). For instance, let $\mu$, resp. $\nu$, be the collection of binary images a sheet of which is depicted on Figure [3(a), resp. Figure 3(b). Then, provided the same membership rule is applied on all the sheets, $\mu(x)=$ $\{\vee\{b, r, g\}, \top\}$ while $\nu(x)=\{r, g, \vee\{b, r, g\}, \top\}$ where $x$ is the one-dimensional face marked on Figure 3. When a point in $\mathbb{F}^{n}$ has not been labeled, for instance,

${ }^{2}$ A formal description of $\mathbb{F}^{n}$ can be found in [1] but no knowledge of cubical complexes is needed to understand the sequel of the article. 
a point in the infinite region surrounding the image, then its fiber is set to $\emptyset$. Since the region obtained by identifying two labels $a$ and $b$, that is, the region associated to the supremum of the labels $a$ and $b$, contains all the faces that are in the region $a$ or in the region $b$ plus, possibly, some other faces (like the one-dimensional face at the center of Figure 1 when we take for $a$ the red label and for $b$ the green one), the fibers are up-sets, that is for any fiber $S$,

$$
t \in S \text { and } t \leq u \Rightarrow u \in S \text {. }
$$

We write $\mathcal{G}_{T}$ for the family of the up-sets over the lattice $T$. Note that $\emptyset \in \mathcal{G}_{T}$.

Eventually, we can define a covering image.

Definition 1 (Covering image). Let $T$ be an atomistic lattice. A covering image $\mu$ is a function from $\mathbb{F}^{n}$ to $\mathcal{G}_{T}$. For any $t \in T$, the sheet $\mu_{t}$ is the binary image defined on $\mathbb{F}^{n}$ by $\mu_{t}(x)=1$ if $t \in \mu(x)$ and $\mu_{t}(x)=0$ otherwise. For any $x \in \mathbb{F}^{n}$, the set $\mu(x)$ is the fiber over $x$.

From Definition 1 and Relation (11), we derive that, for any $t, u \in T$,

$$
t \leq u \Rightarrow \mu_{t} \leq \mu_{u}
$$

where the order on sheets is the pointwise order.

Let $t \in T$ be a label. We set $t^{\uparrow}=\{u \in T \mid t \leq u\}$ and $t^{\downarrow}=\{u \in T \mid u \leq t\}$. The set $\langle t\rangle_{\mu}=\mu_{t}^{-1}(\{1\})$ is the support of $t$ in the covering image $\mu$. Thereby, the expressions $x \in\langle t\rangle_{\mu}, \mu_{t}(x)=1$ and $t \in \mu(x)$ are synonymous. We write $\langle t\rangle_{\mu}^{c}$ for the set $\mathbb{F}^{n} \backslash\langle t\rangle_{\mu}$. The set $\langle T\rangle^{c}=\mu^{-1}(\emptyset)$ is the background of $\mu$. When there is no ambiguity, we write also $\langle t\rangle$ and $\langle t\rangle^{c}$ instead of $\langle t\rangle_{\mu}$ and $\langle t\rangle_{\mu}^{c}$. The support $\langle\perp\rangle$ contains the points in $\mathbb{F}^{n}$ that are in the supports of every labels, in particular every proto-labels. Thus $\langle\perp\rangle$ is empty in a covering image obtain from a digital label image (since a digital label image is a partition of $\mathbb{Z}^{n}$, or a partition of a finite region of $\mathbb{Z}^{n}$ ). Nevertheless, we will need this support to define the dual of a covering image (Section 4): in the dual of a covering image built from a digital label image, the support $\langle\perp\rangle$ is not empty (but so is the background).

Since the lattice $T$ is atomistic, and since, for any set of labels $S$, we have $\left(\bigvee_{t \in S} t\right)^{\uparrow}=\bigcap_{t \in S} t^{\uparrow}$, any element of the codomain $\mathcal{G}_{T}$ of a covering image can be expressed as an union of intersections :

$$
\mathcal{G}_{T}=\left\{\bigcup_{A \in \mathcal{B}} \bigcap_{t \in A} t^{\uparrow} \mid \mathcal{B}: \text { a family of sets of atoms }\right\}
$$

where $\bigcup_{A \in \emptyset} \bigcap_{t \in A} t^{\uparrow}=\emptyset$ and $\bigcap_{t \in \emptyset} t^{\uparrow}=\perp^{\uparrow}=T$. This latter description of $\mathcal{G}_{T}$ gives a way to encode the fibers with trees. 


\section{Simplicity}

A more detailed presentation of the notions of topology used below can be found in our previous work on label images [11.

In a binary image, a point $x$ in the object is simple if its removal from the object "preserves topology" 6]. Since a covering image is a collection of binary images (the sheets), we can extend the notion of simple point to covering images: roughly speaking, in a covering image, a point is simple for a fiber $S$ if it is simple in any sheet modified by the assignment $\mu(x)=S$. In our framework, we use $\beta$-simple points. A point $x \in \mathbb{F}^{n}$ is a $\beta$-simple point for a subset $X$ of $\mathbb{F}^{n}$ if one of the sets $x^{\uparrow} \backslash\{x\}$ or $x^{\downarrow} \backslash\{x\}$ is contractible 3 . The $\beta$-simple points have the advantage to preserve topology twice. Indeed, in the one hand, $\mathbb{F}^{n}$ can be equipped with the Alexandroff topology whose open sets are the up-sets of $\mathbb{F}^{n}$. Then, the deletion of a $\beta$-simple point $x$ from a subset $X$ of $\mathbb{F}^{n}$ is a weak homotopy equivalence, that is, the inclusion $i: X \backslash\{x\} \rightarrow X$ induces a one-to-one correspondence between the connected components of both spaces and induces also isomorphisms between the homotopy groups of $X \backslash\{x\}$ and $X$. Moreover, this is also true for the dual inclusion $i^{\prime}: \mathbb{F}^{n} \backslash X \rightarrow \mathbb{F}^{n} \backslash(X \backslash\{x\})$. On the other hand, one can associate to any subset $X$ of $\mathbb{F}^{n}$ an euclidean set, denoted $|\mathcal{K}(X)|$, which is the realization of a simplicial complex. The deletion from $X$ of a $\beta$-simple point $x$ induces a strong deformation retraction from $|\mathcal{K}(X)|$ to $|\mathcal{K}(X \backslash\{x\})|$. Furthermore, this is also true for the complements (in $\mathbb{R}^{n}$ ) of these realizations but, possibly, in a non-monotonic manner.

Eventually, we can give the definition of a simple point in a covering image.

Definition 2 (Simple point in a covering image). Let $S \in \mathcal{G}_{T}$ be a fiber. $A$ point $x \in \mathbb{F}^{n}$ is simple for (the fiber) $S$ if the following two conditions are verified:

(i) for any label $u \in \mu(x)$ such that $u \notin S, x$ is $\beta$-simple for the set $\langle u\rangle$ or for the set $\langle u\rangle^{c} \cup\{x\}$;

(ii) for any label $u \notin \mu(x)$ such that $u \in S, x$ is $\beta$-simple for the set $\langle u\rangle \cup\{x\}$ or for the set $\langle u\rangle^{c}$.

The previous definition, and the properties of $\beta$-simple points, ensures that, if a point is simple for the fiber $S$ in the image $\mu$, we can set $\mu(x)=S$ while preserving the topology of any region of interest, including the unions pointed out by the choice of the lattice $T$. Moreover, modifications of fibers over points in $\mathbb{F}^{n}$ having the same dimension can be done in parallel, leading to well-balanced algorithms. Figure 4 gives an example of thinning on a covering image using simple points for the fiber $\emptyset$. Observe that in this thinning we have maintained the possibility to go back to $\mathbb{Z}^{n}$ without losing any topological information but this is a difficult issue on which we are still working.

${ }^{3}$ A space is contractible if it has the homotopy type of a point. A subset $X$ of $\mathbb{F}^{n}$ is contractible iff it can be shrunk to a unique point by the sequential removal of unipolar points. A point $x$ is unipolar if $x^{\uparrow} \backslash\{x\}$ has a minimum or $x^{\downarrow} \backslash\{x\}$ has a maximum. 


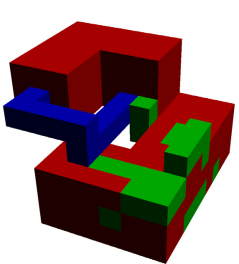

(a)

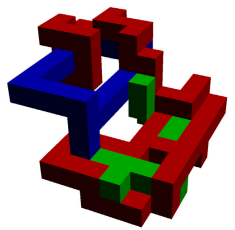

(e)

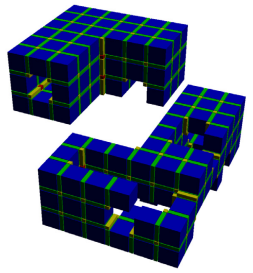

(b)

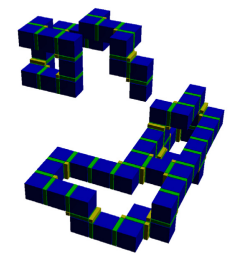

(f)

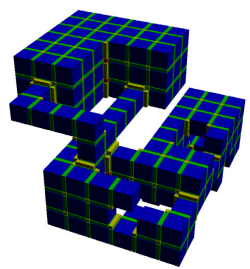

(c)

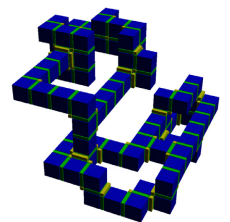

(g)

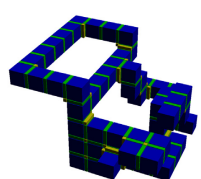

(d)

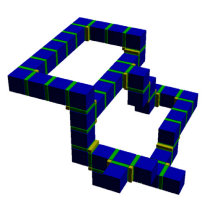

(h)

Fig. 4. (a) A label image $\lambda$ defined on $\mathbb{Z}^{3}$. (b) The "red" sheet $\mu_{\text {red }}$ of the covering image $\mu$ associated to the image $\lambda$ and the $(6,18)$-adjacency pair (on this sheet, which is a binary image, as on the other sheets, the colors are used only to better distinguish the faces of the cubical complex). For any label $t$, and any $f \in \mathbb{F}^{n}$ such that $\operatorname{dim}(f) \leq 2$, the rule used to define the value of $\mu_{t}(f)$ can be expressed as follows: if $\operatorname{dim}(f)=d$ and $f$ bounds at least $k$ black voxels then $f$ is black (i.e. $\mu_{t}(f)=1$ ), otherwise $f$ is white, where $(d, f) \in\{(2,2),(1,3),(0,6)\}$ (see 10]). (c) The "red-or-blue"sheet. (d) The "green-or-blue"sheet. $(\mathrm{e}-\mathrm{h})$ The same as $(\mathrm{a}-\mathrm{d})$ after a thinning procedure (using simple points for the fiber $\emptyset$ ) has been applied. Note that in our implementation we do not directly process the sheets but the fibers (and we extract the sheets for visualization when the process is over).

\section{Duality}

Thanks to the dual order on the lattice $T$ and to the complementation in $2^{T}$, we are able to define the dual of a covering image.

We write $\mathcal{F}_{T}$ for the subset of $2^{T}$ composed of the complements of the elements of $\mathcal{G}_{T}$ in $2^{T}$. As $\mathcal{G}_{T}$ is the family of the up-sets over $T, \mathcal{F}_{T}$ is the family of the down-sets over $T\left(E \in \mathcal{F}_{T}\right.$ iff $e \in E$ and $f \leq e$ imply $\left.f \in E\right)$, i.e. $\mathcal{F}_{T}=\mathcal{G}_{\left(T^{\star}\right)}$ where $T^{\star}$ is the lattice $T$ equipped with its dual order. 
Definition 3 (Dual covering image). The dual covering image of $\mu$ is the covering image $\neg \mu: \mathbb{F}^{n} \rightarrow \mathcal{F}_{T}$ defined for any $x \in \mathbb{F}^{n}$ by $(\neg \mu)(x)=2^{T} \backslash \mu(x)$.

The duality on covering images comes down to a swap of the background and the object in each sheet of a covering image: for any label $t \in T,\langle t\rangle_{\neg \mu}=\langle t\rangle_{\mu}^{c}$ and the background of the dual of a covering image $\mu$ is $\mu^{-1}(T)=\mu^{-1}\left(\perp^{\uparrow}\right)$.

The duality is compatible with the simplicity: a face is simple for a fiber $S$ in a covering image iff it is simple in the dual covering image for the complement in $2^{T}$ of $S$.

Thanks to this duality we can design in one time tools to process digital label images whose regions have to be interpreted with an $(\alpha, \beta)$-adjacency pair or with the $(\beta, \alpha)$-adjacency pair.

\section{Conclusion}

The model exposed in this paper is a way to encompass all the topological relations that characterize a label image. From a theoretical point of view, it can help us to check what is precisely preserved or modified by a procedure. Likely, it is not necessary to implement the model as is. We have begun to look for sufficient conditions to directly process label images defined on $\mathbb{Z}^{3}$ with the conceptual aid of covering images. The first results are tedious but we have not made any optimization on them. On the other hand, it seems predictable that the complexity of a label image (with all the intra-labels and inter-labels relations) is exponentially more costly than the complexity of a single object. Nevertheless, we think that this model can be useful to work on images with several objects when the inter-relations between the objects are meaningful.

Further works will consist in improving the implementation of the whole framework and testing this implementation on "real" images. We plan also to define and study morphological operators for covering images.

\section{References}

1. Ayala, R., Domínguez, E., Francés, A.R., Quintero, A.: Digital Lighting Functions. In: Ahronovitz, E. (ed.) DGCI 1997. LNCS, vol. 1347, pp. 139-150. Springer, Heidelberg (1997)

2. Bazin, P.-L., Ellingsen, L.M., Pham, D.L.: Digital Homeomorphisms in Deformable Registration. In: Karssemeijer, N., Lelieveldt, B. (eds.) IPMI 2007. LNCS, vol. 4584, pp. 211-222. Springer, Heidelberg (2007)

3. Bazin, P.-L., Pham, D.L.: Topology-preserving tissue classification of magnetic resonance brain images. IEEE Transactions on Medical Imaging 26(4), 487-496 (2007)

4. Cointepas, Y., Bloch, I., Garnero, L.: A cellular model for multi-objects multidimensional homotopic deformations. Pattern Recognition 34, 1785-1798 (2001)

5. Damiand, G., Dupas, A., Lachaud, J.-O.: Fully deformable 3D digital partition model with topological control. Pattern Recognition Letters 32, 1374-1383 (2011) 
6. Kong, T.Y., Rosenfeld, A.: Digital topology: Introduction and survey. Computer Vision, Graphics, and Image Processing 48, 357-393 (1989)

7. Latecki, L.J.: Multicolor well-composed pictures. Pattern Recognition Letters 16(4), 425-431 (1995)

8. Liu, J., Huang, S., Nowinski, W.: Registration of brain atlas to MR images using topology preserving front propagation. Journal of Signal Processing Systems 55(1), 209-216 (2009)

9. Mangin, J.-F., Frouin, V., Bloch, I., Régis, J., López-Krahe, J.: From 3D magnetic resonance images to structural representations of the cortex topography using topology preserving deformations. Journal of Mathematical Imaging and Vision 5(4), 297-318 (1995)

10. Mazo, L., Passat, N., Couprie, M., Ronse, C.: Digital imaging: A unified topological framework. Journal of Mathematical Imaging and Vision (to appear, 2012), doi:10.1007/s10851-011-0308-9

11. Mazo, L., Passat, N., Couprie, M., Ronse, C.: Topology on digital label images. Journal of Mathematical Imaging and Vision (to appear, 2012), doi:10.1007/s10851-011-0325-8

12. Miri, S., Passat, N., Armspach, J.-P.: Topology-Preserving Discrete Deformable Model: Application to Multi-segmentation of Brain MRI. In: Elmoataz, A., Lezoray, O., Nouboud, F., Mammass, D. (eds.) ICISP 2008. LNCS, vol. 5099, pp. 67-75. Springer, Heidelberg (2008)

13. Pham, D., Bazin, P.-L., Prince, J.: Digital topology in brain imaging. IEEE Signal Processing Magazine 27(4), 51-59 (2010)

14. Poupon, F., Mangin, J.-F., Hasboun, D., Poupon, C., Magnin, I.E., Frouin, V.: Multi-object Deformable Templates Dedicated to the Segmentation of Brain Deep Structures. In: Wells, W.M., Colchester, A.C.F., Delp, S.L. (eds.) MICCAI 1998. LNCS, vol. 1496, pp. 1134-1143. Springer, Heidelberg (1998)

15. Ronse, C., Agnus, V.: Morphology on label images: Flat-type operators and connections. Journal of Mathematical Imaging and Vision 22(2), 283-307 (2005)

16. Ronse, C., Agnus, V.: Geodesy on label images, and applications to video sequence processing. Journal of Visual Communication and Image Representation 19, 392-408 (2008)

17. Siqueira, S., Latecki, L.J., Tustison, N., Gallier, J., Gee, J.: Topological repairing of 3D digital images. Journal of Mathematical Imaging and Vision 30(3), 249-274 (2008) 